\title{
Neuroblastoma cells can be classified according to glycosphingolipid expression profiles identified by liquid chromatography-tandem mass spectrometry
}

\author{
TOMONORI KANEKO ${ }^{1,2}$, HAJIME OKITA ${ }^{1}$, HIDEKI NAKAJIMA ${ }^{1}$, KAZUTOSHI IIJIMA $^{1}$, \\ NAO OGASAWARA ${ }^{1,2}$, YOSHITAKA MIYAGAWA ${ }^{1}$, YOHKO U. KATAGIRI ${ }^{1}$, ATSUKO NAKAGAWA $^{3}$, \\ NOBUTAKA KIYOKAWA ${ }^{1}$, TOSHINORI SATO ${ }^{2}$ and JUNICHIRO FUJIMOTO ${ }^{4}$
}

${ }^{1}$ Department of Pediatric Hematology and Oncology Research, National Research Institute for Child Health and Development, Setagaya-ku, Tokyo 157-8535; ${ }^{2}$ Department of Biosciences and Informatics, Keio University, Yokohama 223-8522;

${ }^{3}$ Division of Diagnostic Pathology, National Medical Center for Children and Mothers; ${ }^{4}$ Clinical Research Center, National Center for Child Health and Development, Setagaya-ku, Tokyo 157-8535, Japan

Received April 9, 2010; Accepted June 14, 2010

DOI: 10.3892/ijo_00000779

\begin{abstract}
It is hoped that the gangliosides contained in neuroblastomas (NBs) can be used as outcome predictors. We used liquid chromatography-tandem mass spectrometry (LC-MS)

Correspondence to: Dr Nobutaka Kiyokawa, Department of Pediatric Hematology and Oncology Research, National Research Institute for Child Health and Development, 2-10-1, Okura, Setagaya-ku, Tokyo 157-8535, Japan

E-mail: nkiyokawa@nch.go.jp
\end{abstract}

Abbreviations: BSA, bovine serum albumin; $C H G A$, chromogranin A; EIC, extracted ion chromatogram; ESI, Electrospray ionization; GM2, GalNAcß1-4(NeuAca2-3)Galb1-4GlcCer; GD1a, NeuAc $\alpha 2-3$ Galb1-3GalNAcß1-4(NeuAc $\alpha 2-3)$ Galß1-4GlcCer; GD1b, Galß1-3GalNAcß1-4(NeuAc $\alpha 2-8$ NeuAc $\alpha 2-3)$ Galß14GlcCer; GD2, GalNAcß1-4(NeuAc $\alpha 2-8$ NeuAc $\alpha 2-3)$ Galß14GlcCer; GD3, NeuAca2-8NeuAc $\alpha 2-3$ Galß1-4GlcCer; GM1a,

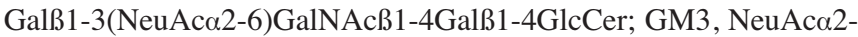
3Galß1-4GlcCer; GQ1b, NeuAca2-8NeuAca2-3Galß1-3GalNAcß14(NeuAc $\alpha 2-8$ NeuAc $\alpha 2-3)$ Galß1-4GlcCer; GT1a, NeuAc $\alpha 2-$ 8NeuAc $\alpha 2-3$ Galß1-3GalNAcß1-4(NeuAc $\alpha 2-3)$ Galß1-4GlcCer; GT1b, NeuAc $\alpha 2-3$ Galß1-3GalNAcß1-4(NeuAc $\alpha 2-8$ NeuAc $\alpha 2-$ 3)Galß1-4GlcCer; Ac-GD2, acetylated GD2; HPTLC, highperformance thin-layer chromatography; HRP, horseradish peroxidase; IT, ion trap; LC-MS, liquid chromatography in combination with mass-spectrometry; mAb, monoclonal antibody; MS/MS, tandem mass spectrometry; MYCN, v-myc myelocytomatosis viral related oncogene; $N$-CAD, $N$-cadherin; $N C A M$, neural cell adhesion molecule; NB, neuroblastoma; NF-H, neurofilament $200 \mathrm{kDa}$ subunit; NF-M, neurofilament $160 \mathrm{kDa}$ subunit; PBS, phosphatebuffered saline; Phox2a, paired-like (aristaless) homeobox $2 a$; Phox $2 b$, paired-like homeobox $2 b$; PTN, pleiotrophin; RT-PCR, reverse transcription-PCR; TrkA, neurotrophic tyrosine kinase, receptor, type 1; TrkC, neurotrophic tyrosine kinase, receptor, type 3

Key words: glycosphingolipid, neuroblastoma, LC-MS to analyze the gangliosides expressed in $11 \mathrm{NB}$ cell lines. LC-MS analysis detected a number of gangliosides, including acetylated forms, with significantly higher sensitivity than conventional high-performance thin-layer chromatography analysis, and the results revealed that the expression profiles of the gangliosides GD1a, GD2, and acetylated GD2 differed according to the NB cell line. Hierarchical clustering based on the ganglioside expression profiles obtained by LC-MS analysis revealed that the NB cell lines could be classified into three types according to their expression of these three gangliosides: A-type characterized by high expression of GD1a and low or no expression of GD2/acetylated GD2, B-type characterized by low or no expression of GD1a and high expression of GD2/acetylated GD2, and AB-type characterized by expression of both GD1a and GD2/acetylated GD2. Interestingly, all three $M Y C N$ non-amplified cell lines were classified into the A-type. The classification was found to be correlated with mRNA expression of ganglioside synthase and neural-differentiation-related genes. The results of this study indicate that LC-MS analysis is useful as a tool for glycosphingolipid research on malignancies.

\section{Introduction}

Ganglioside molecules consist of a sialic acid-containing hydrophilic oligosaccharide chain and a hydrophobic lipid anchor ceramide, and they are embedded in the outer leaflet of the plasma membrane and involved in various cellular processes (1). As summarized in Fig. 1, ganglioside biosynthesis occurs in a series of stepwise glycosylations via two main pathways. One pathway results in the synthesis of GM2, GM1a, GD1a and GT1a (referred to as pathway a in this report), and the other pathway results in the synthesis of GD3, GD2, GD1b, GT1b and GQ1b (referred to as pathway $\mathrm{b}$ in this report). Both pathways proceed from a common precursor, GM3, which is derived from lactosylceramide (2). Analogous steps in biosynthesis pathways a and b are catalyzed by the same glycosyltransferases (Fig. 1) (1). Some 
gangliosides are expressed aberrantly in many types of tumors and are related to the malignant behavior of their cells (3). Research on oligosaccharide chains, however, has not progressed very far because of a lack of effective methods of analyzing oligosaccharide chains. For example, highperformance thin-layer chromatography (HPTLC), a commonly used method to analyze the ganglioside composition of cells, requires large samples and has limited sensitivity for ganglioside detection. Recently improved mass-spectrometry technology, however, may provide an ideal method for analysis of oligosaccharide chains.

Neuroblastomas (NBs) are common solid tumors in childhood and frequently occur in sympathetic ganglia and the adrenal medulla, both of which are derived from the neural crest. The clinical behavior of NBs is known to be varied. The NBs with favorable prognosis often differentiate to mature elements of sympathetic ganglia or regress spontaneously, whereas the NBs with unfavorable prognosis are resistant to chemotherapy, and their long-term outcome remains very poor despite the recent advance in the therapy for NBs. The outcome of NB patients is currently predicted on the basis of a set of risk factors, including age at diagnosis, advanced tumor stage, histological classification according to the international neuroblastoma pathology classification, MYCN amplification, DNA diploidy and chromosomal loss of $1 \mathrm{p}$ in tumors (4). Even when all of these markers are used in combination, it is sometimes difficult to correctly classify the aggressiveness of NBs, and additional markers that can be used as outcome predictors need to be discovered to establish a more effective therapeutic strategy (5).

Previous reports have described a link between expression of certain ganglioside molecules and the behavior of NBs, suggesting that expression of specific gangliosides by NBs may have diagnostic and prognostic potential (6-9). For example, NB cells express a higher level of GD2 than normal brain cells do (10). Characterization of ganglioside expression in the NBs of infants has revealed higher total pathway $b$ ganglioside levels than in older children (8). By contrast, the unfavorable type of NB cells express lower levels of pathway b gangliosides, especially of GD1b, GT1b and GQ1b, than the non-progressive type of NB cells do (9). Based on the above evidence, we are focusing on ganglioside expression as a possible additional marker for predicting the outcome of NB.

In the present study we attempted to determine the overall profile of ganglioside expression in NB cell lines and in greater detail by using liquid chromatography in combination with mass-spectrometry (LC-MS) to determine levels of glycolipid expression. We then classified $11 \mathrm{NB}$ cell lines into three groups based on their ganglioside expression profiles, especially their profiles of GD1a, GD2 and acetylated GD2 expression, and the classification was found to be correlated with the expression of neural-differentiation-related genes.

\section{Materials and methods}

Cell culture and antibodies (Abs). Eleven NB cell lines were used in this study. Nine of the cell lines, GOTO, NB1, NB9, NB16, NB69, IMR32, CHP126, CHP134 and KP-N-NS, were obtained from the Riken Cell Bank (Tsukuba, Ibaraki,

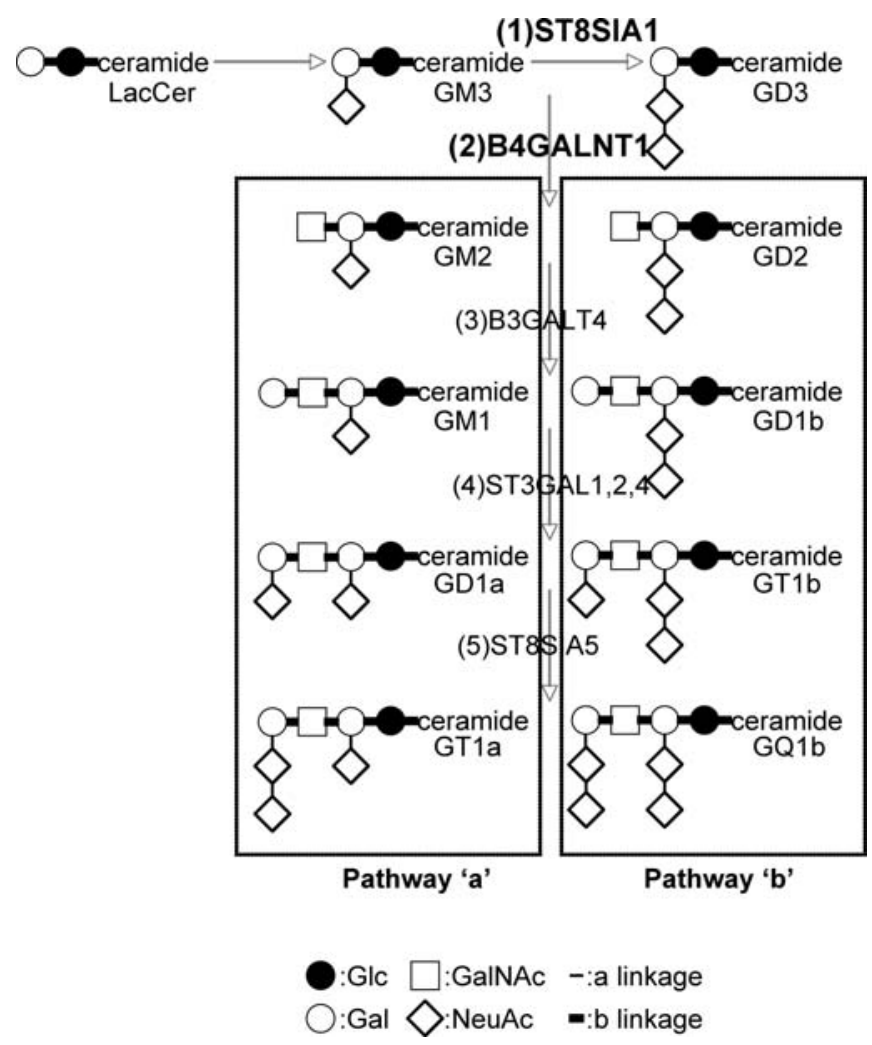

Figure 1. The pathways of ganglioside biosynthesis. The ganglioside biosynthesis pathways and glycosyltransferases that catalyze the synthesis of each ganglioside are shown. The glycosyltransferases whose mRNA expression level was investigated by RT-PCR in this study are indicated in bold type. 1: ST8SIA1, $\alpha 2,8$-sialyltransferase 1 (GD3 synthase); 2: B4GALNT1, B1,4-N-acetylgalactosaminyltransferase 1 (GM2/GD2 synthase); 3: B3GALT4, 31,3-galactosyltransferase 4 (GM1/GD1b synthase); 4: ST3GAL1,2,4, $\alpha 2,3$ sialyltansferase 1,2,4 (GD1a/GT1b synthase); 5: ST8SIA5, $\alpha 2,8$-sialyltransferase 5 (GT1a/GQ1b synthase).

Japan), and the other two cell lines, SK-N-SH and SK-N-RA, were a kind gift from Dr P. Reynolds. The characteristics of the cell lines are summarized in Table I. The MYCN gene is amplified in eight of these cell lines but not in the other three (NB69, SK-N-SH and SK-N-RA). The cells were cultured at $37^{\circ} \mathrm{C}$ in RPMI-1640 (Sigma-Aldrich Co, St. Louis, MO) supplemented with $10 \%$ FBS (Sigma) under a humidified atmosphere of $5 \% \mathrm{CO}_{2}$.

A monoclonal $\mathrm{Ab}(\mathrm{mAb})$ specific for ganglioside GD2, 14.G2a, was purchased from Chemicon (Temecula, CA). Horseradish peroxidase (HRP)-conjugated rabbit anti-mouse immunoglobulin $\mathrm{Ab}$ was purchased from Dako (Glostrup, Denmark).

Lipid extraction. The lipids were extracted from the cell pellet with $2 \mathrm{ml}$ of chloroform/methanol $(2: 1, \mathrm{v} / \mathrm{v})$ and then with $2 \mathrm{ml}$ of chloroform/isopropanol/water (7:11:2, v/v) in a sonicated bath. Total extracts were combined and evaporated to dryness. The lipids extracted from the cells were desalted by a SepPak C18 column (Waters, Milford, MA) and analyzed by HPTLC and LC-MS/MS as described below.

HPTLC analysis. TLC chemical staining and immunostaining were performed according to a previously described method (11). Briefly, the lipids were separated on plates 
Table I. The summary of characteristics of the cell lines.

\begin{tabular}{lllllll}
\hline Cell line & \multicolumn{1}{c}{ Origin } & Age (months) & Sex & Stage & MYCN $^{\text {a }}$ & Author/refs. \\
\hline GOTO & Left adrenal gland & 13 & Male & IV & Amp & Sekiguchi et al (20) \\
NB1 & Neck lymph node & 33 & Male & IV & Amp & Miyake et al (21) \\
NB9 & Adrenal gland & 22 & Male & IV & Amp & Gilbert et al (22) \\
NB16 & Bone marrow & 35 & Female & IV & Amp & Gilbert et al (22) \\
NB69 & Adrenal gland & 16 & Male & III & Not amp & Gilbert et al (22) \\
IMR32 & Abdomen & 13 & Male & IV & Amp & Tumilowicz et al (23) \\
CHP126 & Retroperitoneum & 14 & Female & III & Amp & Schlesinger et al (24) \\
CHP134 & Left adrenal gland & 13 & Male & IV & Amp & Schlesinger et al (24) \\
KP-N-NS & Brain & 10 & Female & IV & Amp & Yoshihara et al $(25)$ \\
SK-N-SH & Bone marrow & 48 & Female & IV & Not amp & Biedler et al (26) \\
SK-N-RA & & & & & Not amp & Helson, unpublished \\
\hline
\end{tabular}

${ }^{a}$ Amplification of MYCN.

precoated with Silica gel 60 (HPTLC sheets, Merck, Darmstadt Germany) by using a solvent system consisting of chloroform/ methanol/water containing $0.2 \% \mathrm{CaCl}_{2}(5: 4: 1, \mathrm{v} / \mathrm{v} / \mathrm{v})$ in duplicate. The HPTLC plates were stained with resorcinol to detect the separated gangliosides. The ganglioside levels were quantitated after scanning the plates on a TLC scanner (model GS-930, Shimadzu). In parallel, other plates were dipped in a $0.1 \%$ polyisobutylmethacrylate (Sigma) cyclohexane solution for $1 \mathrm{~min}$ and blocked with $1 \%$ bovine serum albumin (BSA) in phosphate-buffered saline (PBS). The plates were incubated with the appropriate combination of primary $\mathrm{Ab}$ and HRP-conjugated secondary $\mathrm{Ab}$, and then washed thoroughly. The Abs that bound to the plates were visualized with enhanced chemiluminescence reagent SuperSignal (Pierce, Rockford, IL) and detected by LAS-1000 (Fuji Film, Tokyo, Japan).

Acetylated GD2 (Ac-GD2) was detected as described previously (12). After chromatography, the O-acetyl groups were removed from the gangliosides by exposing the TLC plate to concentrated vapor of ammonium hydroxide in a closed chromatography tank for $12 \mathrm{~h}$, and then allowing the plates to dry. The deacetylated chromatogram was immunostained with anti-GD2 Ab as described above.

LC/ESI-MS analysis. The extracted lipids were separated by high-performance LC (1200 series Capillary LC System, Agilent) equipped with a normal-phase column (Imtakt UKsilica, $150 \times 0.3 \mathrm{~mm})$. Chloroform $/ \mathrm{methanol} / 50 \mathrm{mM}$ acetic acid-triethylamine in water $(\mathrm{pH} 4.2)=83 / 16 / 1(\mathrm{~A})$ and methanol/50 mM acetic acid-triethylamine in water $(\mathrm{pH} 4.2)$ $=3 / 1$ (B) were used as the solvents to determine the lipid composition. Elution was achieved with a linear gradient of $0-100 \%$ of $\mathrm{B}$ over $45 \mathrm{~min}$ at a flow rate of $3 \mu \mathrm{l} / \mathrm{min}$. On-line MS and MS/MS were performed by using an Electrospray ionization (ESI)/ion trap (IT) type mass spectrometer (ESIIT, Bruker Daltonics, Billerica, MA). The lipids were detected by the negative ion mode. The analytical conditions were set to $250^{\circ} \mathrm{C}$ for capillary temperature and $\mathrm{m} / \mathrm{z} 150-2500$ scan range.
Reverse transcription-PCR (RT-PCR) analysis. Total RNA was extracted from cells with an RNeasy Mini Kit (Qiagen, Valencia, CA), and cDNA was generated from $150 \mathrm{ng}$ total RNA by using a FirstStrand cDNA Synthesis Kit (Pharmacia Biotech, Uppsala, Sweden). RT-PCR was performed by using a HotStarTaq Master Mix Kit (Qiagen) according to the manufacturer's protocol. The sets of primers used in this study are listed in Table II. The genes encode enzymes, those involved in gangliosides biosynthesis pathways indicated in Fig. 1, were examined for their expression. The products were separated on a $2 \%$ agarose gel and visualized by ethidium bromide staining.

Clustering analysis. The hierarchical clustering heat map was generated by using package R software (http://www.r-project. $\operatorname{org} /)$.

\section{Results}

Analysis of the composition of the gangliosides in NB cells by $L C$-MS. We established a condition of glycosphingolipid analysis by LC-MS that enables glycosphingolipid expression by cells to be determined. The lipids extracted from NB cells were separated by HPLC and analyzed by MS connected to HPLC on-line. All glycolipid species, the components considered to have ceramide backbones by LCMS detection, were subjected to tandem mass spectrometry (MS/MS), and the molecular species was determined.

The extracted ion chromatogram (EIC) and the mass spectrum of the gangliosides extracted from the CHP134 cells are shown in Fig. 2 and Table III as an example of the results of an analysis. The EIC of the gangliosides and the acetylated gangliosides were superimposed in Fig. 2A and $\mathrm{B}$, respectively. The expression levels of the gangliosides were quantified by calculating the areas of each peak on these chromatograms. Fig. $2 \mathrm{C}$ shows the MS/MS spectrum of the ion at $\mathrm{m} / \mathrm{z} 1645.8$ as a precursor and the structure of the molecular species characterized by the MS/MS fragment ions. The spectrum mainly consists of $\mathrm{Y}$ 
Table II. The sets of primers used in this study.

\begin{tabular}{|c|c|c|}
\hline Name of gene & Forward primer & Reverse primer \\
\hline STSSIAI & TGGGAAATGGTGGGATTCT & TGACAAAGGAGGGAGATTGC \\
\hline B4GALNT1 & GCTGCCTTAGAGCGTTAGACA & GCGAGCAGAAGGACCAGA \\
\hline B3GALT4 & AGGCAGGAACAGGACCTTCT & CCCATATCGCTGTCTTTAGTGAG \\
\hline ST3GAL1 & CAAATCCCGGAAACTCCAG & AGGAAGATGAAATCTGAAAATGGT \\
\hline ST3GAL2 & GTCCAGAGGTGGTGGATGAT & CAGCACCTCATTGGTGTTGT \\
\hline ST3GAL4 & GACCATCCTGAGTGATAAGAAGC & TTAGGATTGACATCCCAGATGA \\
\hline$\beta$-actin & CACCATGTACCCTGGCATT & GCCGATCCACACGGAGTA \\
\hline$N F-M$ & CAGGACCTCCTCAACGTCA & CACCCTCCAGGAGTTTTCTG \\
\hline$N F-H$ & CCGACATTGCCTCCTACC & GGCCATCTCCCACTTGGT \\
\hline NCAM & AGTTTCTCTGCAGGTGGATATTG & GGCATCTCCTGCCACTTG \\
\hline CHGA & GCGGTTTTGAAGATGAACTCTC & GCTCTTCCACCGCCTCTT \\
\hline$p 75$ & GGATCTGATGCTCAAGATGGT & GTCTCTCTCTCTTCACTGGATGG \\
\hline Phox $2 a$ & CACTACCCCGACATTTACACG & GCTCCTGTTTGCGGAACTT \\
\hline Phox $2 b$ & CTACCCCGACATCTACACTCG & CCTGCTTGCGAAACTTGG \\
\hline$M Y C N$ & CCACAAGGCCCTCAGTACC & ССТСТТСАТСАТСТТСАТСАТСТG \\
\hline$p 73$ & ACGTTTGAGCACCTCTGGA & CGCCCACCACCTCATTATT \\
\hline $\operatorname{Trk} A$ & AGGAAGGCCATTCTCTGCTAC & GGCTGAAGTCTTTGGAGAGC \\
\hline $\operatorname{Trk} B$ & GGGACGTGTACAGCACTGACT & CCTGTACATGATGCTCTCTGGA \\
\hline $\operatorname{TrkC}$ & TCTGGGAGATCTTCACCTATGG & CTTGGGTAATGCACTCAATGAC \\
\hline$N$-cadherin & CCTGAAGCCAACCTTAACTGA & TGGAGGGATGACCCAGTCT \\
\hline PTN & AACTGACCAAGCCCAAACCT & GGTGACATCTTTTAATCCAGCA \\
\hline
\end{tabular}

Table III. Fragment ions detected by LC-MS/MS spectra of endogenous GSLs from CHP134 cells.

\begin{tabular}{ll}
\hline Parent $(\mathrm{m} / \mathrm{z})$ & \multicolumn{1}{c}{ Fragments } \\
\hline GM3 $\left(1152.2,[\mathrm{M}-\mathrm{H}]^{-}\right)$ & $1133.6,860.5,698.4,680.4,536.4,518.4$ \\
GM2 $\left(1355.5,[\mathrm{M}-\mathrm{H}]^{-}\right)$ & $1339.4,1156.6,1063.6,860.5,698.5,680.4,536.5$ \\
GM1 $\left(1517.2,[\mathrm{M}-\mathrm{H}]^{-}\right)$ & $1498.5,1456.6,1438.6,1225.6,1063.6,1045.6,860.6,698.5,680.4,536.5$ \\
GD1a $\left(903.9,[\mathrm{M}-2 \mathrm{H}]^{2-}\right)$ & $1516.6,1498.6,1225.5,1151.4,1063 . .5,997.5,860.5,680.4,536.6,290.0$ \\
GT1a $\left(1049.6,[\mathrm{M}-2 \mathrm{H}]^{2-}\right)$ & $1810.4,1516.5,1226.4,903.2,581.1,537.2$ \\
GD3 $\left(721.4,[\mathrm{M}-2 \mathrm{H}]^{2-}\right)$ & $1151.5,1133 . .6,860.5,698.5,581.1,537.4,290.0$ \\
GD2 $\left(822.9,[\mathrm{M}-2 \mathrm{H}]^{2-}\right)$ & $1354.6,1337.6,1063.6,860.5,698.5,680.4,581.1,536.4,290.0$ \\
GD1b $\left(904.0,[\mathrm{M}-2 \mathrm{H}]^{2-}\right)$ & $1516.6,1498.6,1225.6,1064.6,1046.4,997.5,860.5,698.5,680.4,581.1,536.5,290.0$ \\
GT1b $\left(1049.6,[\mathrm{M}-2 \mathrm{H}]^{2-}\right)$ & $1807.5,1789.4,1516.7,1499.6,1225.5,1064.666,903.8,860.6,680.4,581.1,537.2,290.1$ \\
AcGD1a $\left(925,[\mathrm{M}-2 \mathrm{H}]^{2-}\right)$ & $1558.4,1516.6,1225.6,1151.8,1064.7,860.6,698.4,680.0,536.4,332.2,290.0$ \\
Ac-GD3 $\left(743.0,[\mathrm{M}-2 \mathrm{H}]^{2-}\right)$ & $1151.5,860.5,698.4,680.4,623.2,536.5,332.0,290.0$ \\
Ac-GD2 $\left(843.9,[\mathrm{M}-2 \mathrm{H}]^{2-}\right)$ & $1354.6,1063.6,860.5,698.5,680.4,623.1,536.5,332.2,290.0$ \\
Ac-GD1b $\left(925.1,[\mathrm{M}-2 \mathrm{H}]^{2-}\right)$ & $1516.5,1225.6,1063.5,1045.6,860.5,698.5,623.1,536.5,332.1,290.0$ \\
Ac-GT1b $\left(1070.6,[\mathrm{M}-2 \mathrm{H}]^{2-}\right)$ & $1516.5,1048.2,623.6$ \\
A (758.4, $\left.[\mathrm{M}-2 \mathrm{H}]^{2-}\right)$ & $1225.5,1063.4,860.4,698.4,536.5,290.1$ \\
B (860.0, $\left.[\mathrm{M}-2 \mathrm{H}]^{2-}\right)$ & $1428.5,1063.6,1038.2,997.4,876.1,698.6,673.1,536.4,289.9$ \\
C (940.8, $\left.[\mathrm{M}-2 \mathrm{H}]^{2-}\right)$ & $1590.5,1429.6,1411.6,1225.5,1063.5,860.6,698.6,536.4,290.0$ \\
\hline
\end{tabular}

ions, i.e., the $\mathrm{Y} 3 \alpha$ at $\mathrm{m} / \mathrm{z} 1354.6$ (loss of outer sialic acid), $\mathrm{Y} 2 \alpha$ at $\mathrm{m} / \mathrm{z} 1063.6$ (loss of inner sialic acid), $\mathrm{Y} 2 \alpha / \mathrm{Y} 2 \beta$ at $\mathrm{m} / \mathrm{z}$
860.5 (loss of outer GalNAc), Y1 at m/z 698.5 (Lac-Cer) and $\mathrm{Y} 0$ at $\mathrm{m} / \mathrm{z} 536.5$ [Cer, the sphingosine (d18:1) with 
A
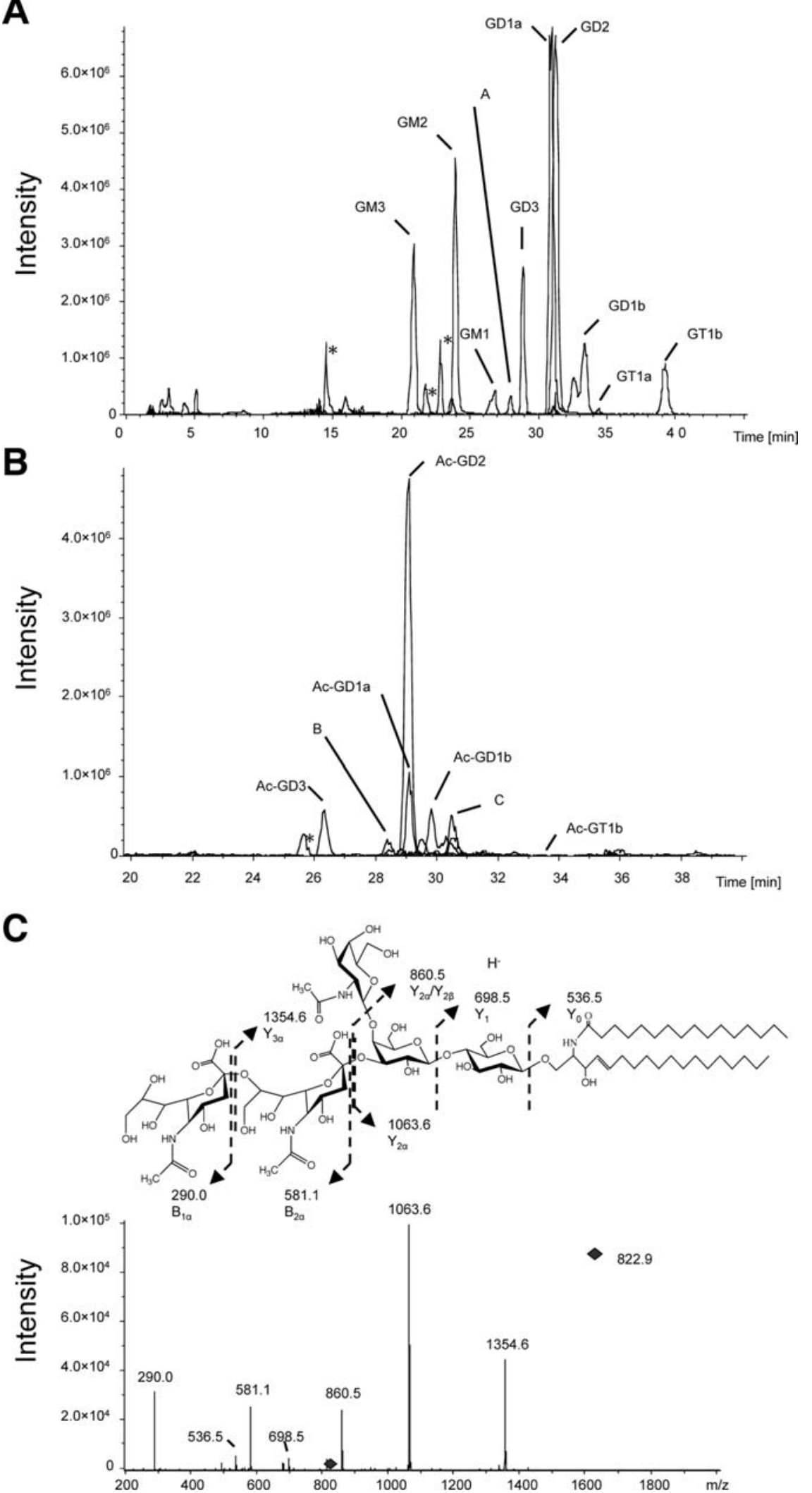

Figure 2. The LC-MS analysis of gangliosides extracted from the CHP134 cells. (A) Superimposed EIC of GM3, GM2, GM1, peak A, GD3, GD1a, GD2, GD1b, GT1a and GT1b extracted from 2x106 of CHP134 cells. (B) Superimposed EIC of Ac-GD3, peak B, Ac-GD1a, Ac-GD2, Ac-GD1b, peak C and Ac-GT1b extracted from $2 \times 10^{6}$ of CHP134 cells. Asterisks indicate the impurity peak that did not represent molecular species of ceramide. (C) The MS/MS spectrum of the ion at $\mathrm{m} / \mathrm{z} 822.9[\mathrm{M}-2 \mathrm{H}]^{2-}$ as a precursor and the chemical structure of the molecular species characterized by the MS/MS fragment ions. All fragment ions appeared as singly charged species.

C16:0 fatty acid] (Fig. 2C). Two B ions, i.e., B1 $\alpha$ at $\mathrm{m} / \mathrm{z} 290.0$ (Neu5Ac) and B2 $\alpha$ at $\mathrm{m} / \mathrm{z} 581.1$ (Neu5Ac-Neu5Ac), were also detected (Fig. 2C). Since these fragment patterns corresponded to those of standard GD2 (data not shown), the 
Table IV. Ganglioside composition of human neuroblastoma cell lines (Cer: d 18:1/16:0).

\begin{tabular}{|c|c|c|c|c|c|c|c|c|c|c|c|}
\hline & \multicolumn{11}{|c|}{$\%$ of total gangliosides } \\
\hline & SK-N-SH & SK-N-RA & NB69 & GOTO & NB9 & CHP134 & KP-N-NS & IMR32 & NB1 & NB16 & CHP126 \\
\hline GM3 & 44.44 & 17.04 & 11.05 & 13.02 & 6.43 & 7.28 & 4.00 & 11.50 & 13.19 & 18.11 & 4.96 \\
\hline GM2 & 9.93 & 8.63 & 22.19 & 31.77 & 21.55 & 11.51 & 11.34 & 31.31 & 23.41 & 17.75 & 20.54 \\
\hline GM1 & 9.72 & 11.04 & 12.86 & 4.43 & 4.80 & 8.22 & 6.56 & 5.52 & 4.63 & 8.30 & 4.20 \\
\hline GD1a & 29.13 & 33.38 & 23.30 & 33.53 & 53.29 & 19.31 & 21.14 & 7.62 & 5.20 & 7.01 & 8.40 \\
\hline GT1a & 0.00 & 0.00 & 0.00 & 0.00 & 0.00 & 0.15 & 0.27 & 0.00 & 0.00 & 0.00 & 0.00 \\
\hline GD3 & 0.48 & 13.23 & 1.35 & 2.05 & 2.71 & 6.17 & 3.99 & 3.67 & 4.61 & 4.23 & 3.75 \\
\hline GD2 & 0.10 & 3.97 & 4.25 & 7.59 & 3.77 & 21.43 & 23.94 & 21.88 & 26.60 & 25.99 & 37.11 \\
\hline GD1b & 0.47 & 5.38 & 7.29 & 0.00 & 0.00 & 6.21 & 12.89 & 0.45 & 0.62 & 2.25 & 7.36 \\
\hline GT1b & 0.04 & 1.52 & 0.10 & 0.14 & 0.51 & 2.94 & 3.40 & 0.12 & 0.09 & 0.45 & 0.93 \\
\hline AcGD1a & 0.52 & 0.61 & 0.56 & 1.30 & 1.77 & 1.46 & 1.61 & 0.28 & 0.28 & 0.42 & 0.33 \\
\hline AcGD3 & 0.00 & 0.71 & 0.00 & 0.07 & 0.00 & 1.15 & 0.00 & 0.33 & 1.24 & 0.26 & 0.28 \\
\hline AcGD2 & 0.00 & 0.77 & 0.66 & 1.48 & 0.65 & 7.83 & 7.71 & 9.17 & 13.78 & 12.07 & 9.17 \\
\hline AcGD1b & 0.00 & 0.00 & 0.00 & 0.00 & 1.02 & 1.53 & 1.35 & 0.41 & 0.45 & 0.81 & 0.83 \\
\hline AcGT1b & 0.00 & 0.00 & 0.00 & 0.00 & 0.00 & 0.03 & 0.00 & 0.00 & 0.00 & 0.00 & 0.00 \\
\hline A & 0.00 & 0.00 & 5.95 & 0.99 & 0.41 & 3.87 & 0.51 & 1.84 & 2.04 & 0.00 & 0.00 \\
\hline B & 3.09 & 0.53 & 1.93 & 0.22 & 0.49 & 0.00 & 0.62 & 0.70 & 0.34 & 0.77 & 1.46 \\
\hline $\mathrm{C}$ & 2.08 & 3.18 & 8.49 & 3.40 & 2.60 & 0.93 & 0.65 & 5.20 & 3.53 & 1.57 & 0.69 \\
\hline
\end{tabular}

A, NeuAc-Hex+HexNAc+LacCer; B, NeuAc+HexNAc+(Gal-GlcNAc)+LacCer; C, NeuAc+(Gal-GlcNAc $)_{2}+$ LacCer.

structure of the ion at $\mathrm{m} / \mathrm{z} 822.9[\mathrm{M}-2 \mathrm{H}]^{2-}$ was identified as that of GD2.

Fragment ions observed by LC-MS/MS spectra of each peak in Fig. 2A and B and the deduced molecular species were listed in Table III. The sugar composition of peak A, B and $\mathrm{C}$ did not correspond to ganglio-series gangliosides, and the structure was predicted as NeuAc-Hex+HexNAc+ LacCer, NeuAc+HexNAc+Hex-HexNAc+LacCer and $\mathrm{NeuAc}+(\mathrm{Hex}-\mathrm{HexNAc})_{2}+\mathrm{LacCer}$, respectively.

The composition of the individual gangliosides in each cell line was expressed as a percentage of its peak area to the total area of all of the ganglioside peaks. Since each ganglioside carried at least two major ceramides, $d$ 18:1/16:0 and d $18: 1 / 24: 1$, a semi-quantitative value of each ganglioside was calculated for each major ceramide having $\mathrm{m} / \mathrm{z} 535.6$ and 646.6, and the values are summarized in Table IV (d 18:1/16:0) and Table V (d 18:1/24:1). It should be noted that several gangliosides also carried a few minor ceramide species (d 18:1/18:0, d 18:1/20:0, d 18:1/22:0) (data not shown).

As shown in Tables IV and V, expression of GD1a, one of the pathway a ganglioside, was high in SK-N-SH, SK-NRA, NB69, GOTO, NB9, CHP134 and KP-N-NS cells and low in IMR32, NB1, NB16 and CHP126 cells, whereas expression of GD2, a pathway b ganglioside, was high in CHP134, KP-N-NS, IMR32, NB1, NB16 and CHP126 cells and low in SK-N-SH, SK-N-RA, NB69, GOTO and NB9 cells.

Interestingly, the gangliosides containing an $\mathrm{O}$-acetylated sialic acid were detected in all of the NB cell lines (Tables IV and V). Acetylation was observed in GD1a, GD3, GD2, GD1b and GT1b, but the proportion of the acetylated-gangliosides varied from cell line to cell line. Expression of Ac-GD2 was high in CHP134, KP-N-NS, IMR32, NB1, NB16 and CHP126 cells, accounting for $>10 \%$ of all gangliosides in NB1 and NB16 cells, and low in SK-N-SH, SK-N-RA, NB69, GOTO and NB9 cells (Tables IV and V).

Analysis of ganglioside composition by HPTLC. To confirm the reliability of the profiles of ganglioside expression in NB cells obtained by LC-MS, samples of the same lipid extract preparations were analyzed by HPTLC, and the relative amounts of the individual gangliosides they contained were determined by densitometry (Fig. 3, Table VI). Nine bands stained with resorcinol were tentatively assigned to GM3, GM2, GM1, GD3, GD2, GD1a, GD1b, GT1a and GT1b, respectively, by comparing the $\mathrm{Rf}$ values with ganglioside standards. All of them were also detected by LC-MS and the expression profiles of these gangliosides were highly consistent with those detected by LC-MS. However, the other gangliosides, including acetylated forms, that were identified by LC-MS were not detected by HPTLC. For example, HPTLC could not detect GD2 in SK-N-SH, SK-N-RA, NB69, GOTO and NB9, although its expression in the cells was detected by LC-MS.

GD2 and Ac-GD2 ganglioside detected by TLC immunostaining. We performed a TLC immunostaining analysis to confirm the differences in the level of GD2 expression among the NB cell lines. As shown in Fig. 4A, the lipid extracts from SK-N-SH, SK-N-RA, NB69, GOTO and NB9 cells were weakly or hardly stained with anti-GD2 mAb 14.G2a (lanes 1-5), whereas those of CHP-134, KP-N-NS, IMR32, 


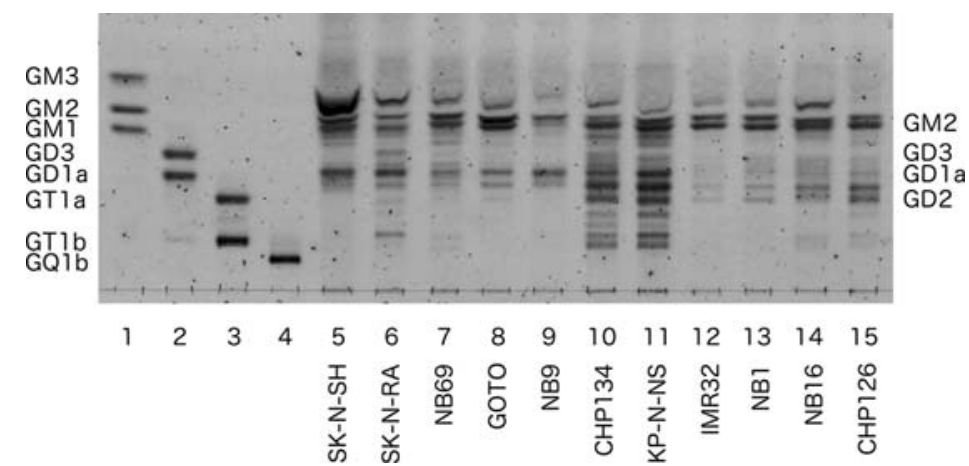

Figure 3. High-performance thin-layer chromatogram of gangliosides extracted from the human neuroblastoma cell lines. Lipids extracted from $1 \times 10^{7}$ cells were separated on an HPTLC plate and visualized by resorcinol spraying. GM3, GM2, GM1, GD3, GD1a, GT1a, GT1b and GQ1b were used as ganglioside standards (lanes 1-4).

Table V. Ganglioside composition of human neuroblastoma cell lines Cer: d 18:1/24:1.

\begin{tabular}{|c|c|c|c|c|c|c|c|c|c|c|c|}
\hline & \multicolumn{11}{|c|}{$\%$ of total gangliosides } \\
\hline & SK-N-SH & SK-N-RA & NB69 & GOTO & NB9 & CHP134 & KP-N-NS & IMR32 & NB1 & NB16 & CHP126 \\
\hline GM3 & 36.89 & 6.22 & 3.41 & 4.90 & 4.80 & 1.20 & 0.49 & 3.05 & 4.88 & 7.88 & 0.60 \\
\hline GM2 & 8.40 & 17.05 & 28.82 & 15.26 & 5.93 & 6.79 & 8.08 & 24.80 & 19.21 & 12.06 & 15.25 \\
\hline GM1 & 7.11 & 13.94 & 4.6 & 7.24 & 9.65 & 6.86 & 5.43 & 5.19 & 3.6 & 6.52 & 2.44 \\
\hline GD1a & 37.09 & 40.36 & 45.55 & 57.80 & 35.00 & 29.28 & 26.75 & 14.56 & 8.48 & 11.58 & 13.64 \\
\hline GT1a & 0.00 & 0.00 & 0.00 & 0.13 & 0.00 & 0.25 & 0.54 & 0.00 & 0.00 & 0.00 & 0.34 \\
\hline GD3 & 0.00 & 1.34 & 0.79 & 0.83 & 20.53 & 8.10 & 3.92 & 3.53 & 4.89 & 4.70 & 2.62 \\
\hline GD2 & 0.00 & 2.28 & 6.70 & 2.37 & 3.83 & 13.05 & 10.14 & 25.34 & 27.55 & 27.02 & 40.60 \\
\hline GD1b & 1.66 & 0.00 & 0.00 & 0.00 & 7.11 & 6.96 & 19.07 & 0.89 & 2.03 & 3.27 & 0.00 \\
\hline GT1b & 0.00 & 0.20 & 0.18 & 0.87 & 2.20 & 3.00 & 3.35 & 0.00 & 0.00 & 0.61 & 2.08 \\
\hline AcGD1a & 3.04 & 1.29 & 3.04 & 4.56 & 1.26 & 2.46 & 2.61 & 0.70 & 0.50 & 0.74 & 1.01 \\
\hline AcGD3 & 0.00 & 0.00 & 0.00 & 0.00 & 0.00 & 1.07 & 0.00 & 0.31 & 0.97 & 0.81 & 0.00 \\
\hline AcGD2 & 0.00 & 0.58 & 2.64 & 1.04 & 1.10 & 11.07 & 10.71 & 14.15 & 21.59 & 20.25 & 16.42 \\
\hline AcGD1b & 0.00 & 0.00 & 0.00 & 0.78 & 3.68 & 3.19 & 6.81 & 0.00 & 0.92 & 1.75 & 2.54 \\
\hline AcGT1b & 0.15 & 0.00 & 0.00 & 0.00 & 0.14 & 1.68 & 0.00 & 0.00 & 0.00 & 0.00 & 0.00 \\
\hline A & 0.00 & 6.19 & 1.22 & 0.94 & 0.00 & 3.12 & 0.53 & 1.64 & 1.55 & 0.00 & 0.00 \\
\hline B & 3.97 & 2.68 & 0.00 & 0.79 & 1.38 & 0.67 & 0.82 & 1.04 & 0.57 & 0.77 & 1.70 \\
\hline $\mathrm{C}$ & 1.67 & 7.88 & 3.04 & 2.48 & 3.38 & 1.26 & 0.77 & 4.81 & 3.27 & 2.05 & 0.74 \\
\hline
\end{tabular}

A, [NeuAc-Hex+HexNAc+LacCer; B, [NeuAc+HexNAc+(Gal-GlcNAc)+LacCer; C, [NeuAc+(Gal-GlcNAc) $)_{2}+\mathrm{LacCer}$.

Table VI. Ganglioside composition of human neuroblastoma cell lines (HPTLC).

\begin{tabular}{lrrrrrrrrrrr}
\hline & \multicolumn{10}{c}{ \% of total gangliosides } \\
\cline { 2 - 11 } & SK-N-SH & SK-N-RA & NB69 & GOTO & NB9 & CHP134 & KP-N-NS & IMR32 & NB1 & NB16 & CHP126 \\
\hline GM3 & 52.45 & 20.65 & 17.56 & 15.01 & 7.34 & 5.92 & 3.40 & 11.66 & 14.09 & 17.40 & 7.18 \\
GM2 & 25.36 & 29.50 & 49.09 & 59.76 & 42.39 & 18.83 & 19.27 & 63.44 & 49.54 & 35.99 & 34.89 \\
GM1 & 1.68 & 1.12 & 4.84 & 1.98 & 0.51 & 7.81 & 8.87 & 2.99 & 3.80 & 4.31 & 5.24 \\
GD1a & 20.50 & 29.27 & 19.86 & 20.51 & 49.75 & 16.06 & 13.97 & & 2.17 & 6.60 & 0.48 \\
GT1a & & 2.54 & 0.28 & 0.60 & & 6.70 & 8.85 & & & & \\
GD3 & & 9.26 & 6.35 & 2.13 & & 5.86 & 5.30 & 10.40 & 15.08 & 9.81 & 11.91 \\
GD2 & & & & & & 15.86 & 15.21 & 11.52 & 15.33 & 21.86 & 34.22 \\
GD1b & & & & & & 12.18 & 11.92 & & & 0.56 & 2.04 \\
GT1b & 7.66 & 2.03 & & & 10.78 & 13.22 & & & 3.47 & 4.05 \\
\hline
\end{tabular}


A

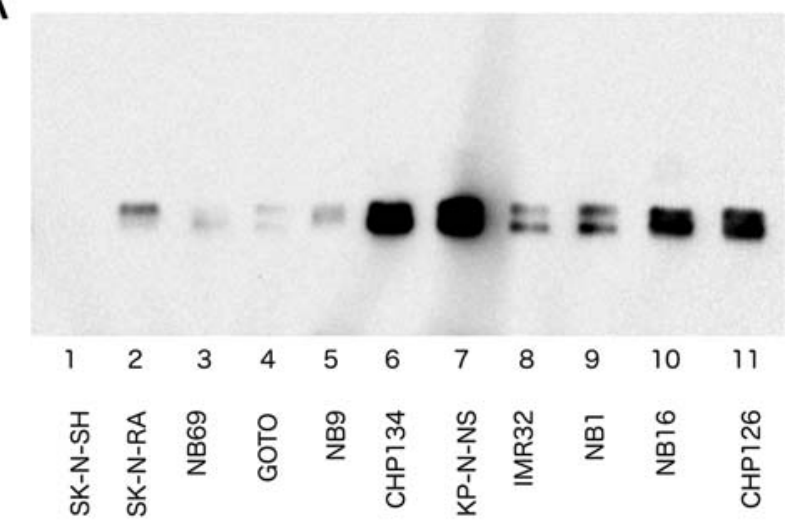

B

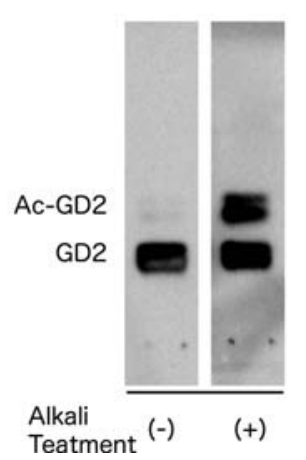

Figure 4. TLC immunostaining of ganglioside GD2 and Ac-GD2. (A) The lipids extracted from $1 \times 10^{5}$ cells were separated on HPTLC plates and immunostained with mAb 14.G2a. (B) The lipids of $1 \times 10^{5}$ CHP134 cells separated on an HPTCL plate was treated with alkali and immunostained with mAb 14.G2a.

NB1, NB16 and CHP126, were strongly or significantly stained (lanes 6-11). These results are essentially consistent with those obtained with LC-MS.

As shown in Fig. 4B, when the TLC plate was treated with alkali after TLC development to remove acetyl group from sialic acid residues, the band newly produced by the conversion of Ac-GD2 to GD2 was detected at a position with a higher Rf value than GD2. Taken together with the results of the LC-MS analysis, these findings show that AcGD2 is expressed in NB cells.

Clustering analysis of gangliosides expressed in NB cells. The above results imply that the NB cell lines have distinct glycolipid expression profiles that allow them to be classified in several groups. As shown in Tables IV, V and Fig. 5, the profiles of glycosphingolipid GD1a, GD2 and acetylated GD2 expression varied from cell line to cell line. We therefore performed a clustering analysis centered on these three glycosphingolipids, and the results showed that the NB cell lines could be classified into three groups based on their expression of these three gangliosides (Fig. 5B). The NB9, NB69, SK-N-SH, SK-N-RA and GOTO cells were characterized by high expression of GD1a and low expression of GD2/acetylated GD2 and classified as type A here, whereas CHP126, IMR32, NB1 and NB16 cells were characterized by low expression of GD1a and high expression of GD2/ acetylated GD2 and classified as type B. CHP134 and KP-NNS cells, on the other hand, were characterized by expression of both GD1a and GD2/acetylated GD2 and classified as type $\mathrm{AB}$. Interestingly, all three $M Y C N$ non-amplified cell lines were classified as type A.

Expression of ganglioside synthase mRNA detected by RT$P C R$ in NB cell lines. To investigate the relationship between ganglioside expression and the level of expression of enzymes involved in ganglioside biosynthesis, RT-PCR analysis was used to investigate the NB cell lines for mRNA expression of two ganglioside synthases. As shown in Fig. 6A, the results showed a high level of GD3 synthase mRNA expression (ST8SIA1) (Fig. 1) in the types B and AB cell lines, whereas mRNA expression of B4GALNT1, which is responsible for catalyzing the synthesis of both GM2 and GD2 (Fig. 1), was detected in all of the cell lines.

RNA expression of neural differentiation markers detected by $R T-P C R$ in NB cell lines. To investigate the biological significance of the classification of NB cell lines based on their ganglioside expression profile, we investigated expression of neural-differentiation-related genes by RT-PCR and assessed the relation between their expression and glycolipid expression. As shown in Fig. 6B and C, RT-PCR analysis revealed that mRNA expression of the neural-differentiationrelated genes Phox $2 a$ and $b, T r k C$, neurofilament, and $N-C A M$ was positively correlated with GD2 and acetylated GD2 expression in the NB cell lines. By contrast, mRNA expression of pleiotrophin $(P T N)$ tended to be high in cell lines with a low level of GD2 expression. All MYCN non-amplified cells show low MYCN expression by RT-PCR (Fig. 6D).

\section{Discussion}

In this study we used an LC-MS analysis system to detect glycosphingolipid expression in NB cells and clearly demonstrated its great potential as a tool for glycosphingolipid research. As shown above, the LC-MS analysis was highly sensitive and enabled detection of a number of glycolipids expressed in NB cells that were not detected by HPTLC analysis. This approach allows determination of even low percentages of lipids of each molecular species and showed clear differences between the glycosphingolipid profiles of a series of NB cell lines. The method described in this report should be useful and easily adaptable to glycosphingolipid analysis of various types of tumor cells.

In this study we also demonstrated the presence of acetylated forms of gangliosides in NB cells. As described above, we detected acetylated forms of GD1a, GD3, GD2, GD1b and GT1b in NB cells by LC-MS analysis and expression of acetylated GD2 was found to be high. For example, Ac-GD2 accounted for $>10 \%$ of the total ganglioside in NB1 and NB16 cells. The presence of acetylated GD2 was correlated with expression of GD2. Ye and Cheung demonstrated that $\mathrm{O}$-acetylated GD2 is a naturally occurring ganglioside derivative in human tumors, including in NB, by using mAb 3F8, which specifically recognizes GD213, and our data are consistent with their findings. 
A
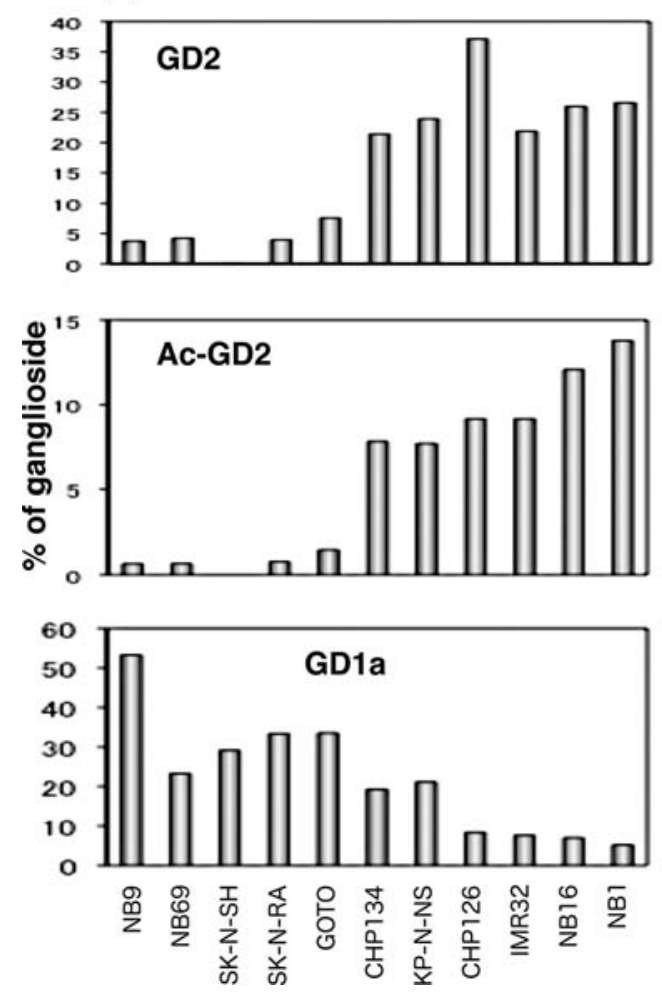

B

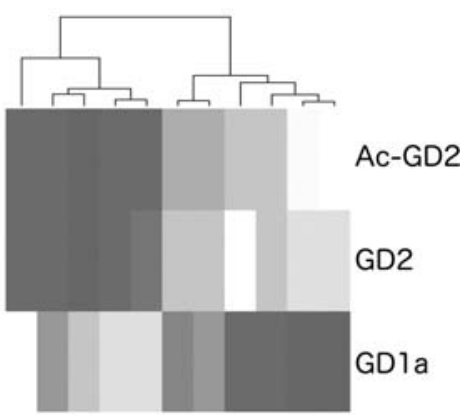



Figure 5. Hierarchical clustering of differentially expressed gangliosides. (A) The percentage of Ac-GD2, GD2 and GD1a to the total gangliosides of NB cell lines. (B) The clustering tree shows the expression pattern and similarity in cell lines. The strength of the gangioside expression was gradually increased on the heat map.

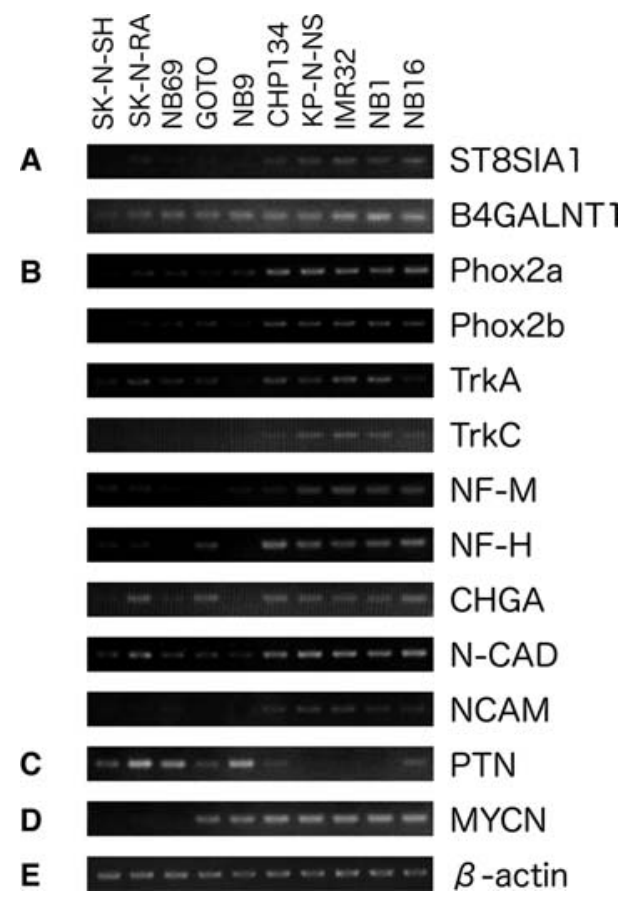

Figure 6. Analysis of expression of neural-differentiation-related genes and glycosyltransferase genes by RT-PCR. (A) Glycosyltransferase genes (Fig. 1), (B) Phox $2 a$, paired-like (aristaless) homeobox 2a; Phox $2 b$, paired-like homeobox 2b; $\operatorname{Trk} A$, neurotrophic tyrosine kinase, receptor, type 1, also known as NTRK1; TrkC, neurotrophic tyrosine kinase, receptor, type 3, also known as NTRK3; $N F-M$, neurofilament 160 kDa subunit; $N F-H$, neurofilament $200 \mathrm{kDa}$ subunit; $C H G A$, chromogranin A; N-CAD, N-cadherin and NCAM, neural cell adhesion molecule, (C) PTN, pleiotrophin, (D) MYCN, $\mathrm{v}$-myc myelocytomatosis viral related oncogene, (E) $\beta$-actin was used as an internal control.
The biological significance of ganglioside acetylation has not been fully elucidated, but it is thought to modulate cell function by regulating the ability of gangliosides to bind cell adhesion molecules. For example, CD22ß (also called Siglec-2) is a B-cell-restricted phosphoprotein that mediates interactions with other cells via binding with a2-6-linked sialic acids on glycoconjugates, and the fact that the binding can be inhibited by 9-O-acetylation of sialic acids suggests that $\mathrm{CD} 22 \beta$ adhesion events are regulated by ganglioside acetylation $(14,15)$. In childhood acute lymphoblastic leukemia, on the other hand, administration of exogenous GD3 induces apoptosis, whereas O-acetylated GD3 fails to induce similar effects, suggesting that O-acetylation of GD3 promotes leukemia cell survival by preventing apoptosis $(16,17)$. Although the significance of acetylated GD2 in NB cells still remains largely unknown, further investigation should shed light on the functional role of gangliosides in the biological behavior of NB cells.

The NB cell lines were classified into three types based on their of ganglioside expression profiles determined by LCMS analysis, namely, type A, with a high level of expression of GD1a but low level or no expression of GD2/acetylated GD2, and consisting of SK-N-SH, SK-N-RA, NB69, GOTO and NB9 cells, type B, with a high level of expression of GD2/acetylated GD2 but low level or no expression of GD1a, and consisting of IMR32, NB1, NB16 and CHP126 cells, and type $\mathrm{AB}$, which express both GD1a and GD2/acetylated GD2, and consisting of CHP134 and KP-N-NS cells. The results of the RT-PCR analyses indicated that the ganglioside expression profiles of NBs correlated with their ganglioside 
synthase expression pattern. As shown in Fig. 6, ST8sia1, which catalyzes the synthesis of GD3 from GM3, was expressed only in the types $\mathrm{B}$ and $\mathrm{AB}$ NB cell lines and not in any of the type A NB cell lines, whereas B4galnt1, which catalyzes the synthesis of both GM2 and GD2, was expressed in all the NB cell lines tested in this study.

Expression of GD2 ganglioside is characteristic of cells of neuroectodermal origin, and a high level of expression has been reported in NB cells, whereas the GD2 distribution in humans is limited to neurons and peripheral nerve fibers (18). Thus, GD2 appears to be useful as a target for the treatment of NB. However, our findings in this study indicated that the level of GD2 expression in NB cells is variable and that NB cells can be classified based on their pattern of expression of ganglio-series gangliosides, including GD2. Since increased shedding of GD2 ganglioside and MYCN amplification jointly characterize the aggressive type of NB cells (19), classification of NBs based on their ganglioside expression profile may have prognostic value. Our observation that the ganglioside expression profiles are closely related to the expression of neural-differentiation-related genes appears to further support this notion.

In conclusion, we have demonstrated the usefulness of the LC-MS analysis system as a tool for glycosphingolipid research. Eighteen species of glycosphingolipids containing gangliosides of $\mathrm{a}$ and $\mathrm{b}$ pathways and their acetylated forms were detected. The expression ratios of the glycosphingolipids were determined, and were compared among 11 of NB cell lines. Based on the results, it was indicated that these NB cell lines could be classified into three categories. Although more detailed experiments are clearly needed, further investigations using the new method should provide a new approach to determining the biological significance of glycosphingolipids in NBs and identifying novel biomarkers for predicting the outcome of NB.

\section{Acknowledgments}

We are grateful to Dr P. Reynolds for providing the SK-NSH and SK-N-RA cells. We thank Ms. H. Kiyokawa for her assistance to prepare the manuscript. This work was supported by a grant from the Japan Health Sciences Foundation for Research on Publicly Essential Drugs and Medical Devices (KHA1004), Health and Labour Sciences Research Grants (Research on Human Genome Tailor made and Research on Publicly Essential Drugs and Medical Devices H18-005, the 3rd-term Comprehensive 10-year strategy for Cancer Control H19-010), a Grant for Child Health and Development from the Ministry of Health, Labour and Welfare of Japan, and by CREST, JST. This work was supported in part by the Grantin-Aid for Cancer Research (16-16) from the Ministry of Health, Labor and Welfare.

\section{References}

1. Ledeen RW and Yu RK: Gangliosides: structure, isolation, and analysis. Methods Enzymol 83: 139-191, 1982.

2. Van Echten G and Sandhoff K: Ganglioside metabolism. Enzymology, topology, and regulation. J Biol Chem 268: 5341-5344, 1993.

3. Hakomori S: Tumor malignancy defined by aberrant glycosylation and sphingo(glyco)lipid metabolism. Cancer Res 56: 5309-5318, 1996.
4. Weinstein JL, Katzenstein HM and Cohn SL: Advances in the diagnosis and treatment of neuroblastoma. Oncologist 8: 278-292, 2003.

5. Ohira M, Oba S, Nakamura Y, Hirata T, Ishii $S$ and Nakagawara A: A review of DNA microarray analysis of human neuroblastomas. Cancer Lett 228: 5-11, 2005.

6. Schengrund CL, Repman MA and Shochat SJ: Ganglioside composition of human neuroblastomas. Correlation with prognosis. A Pediatric Oncology Group Study. Cancer 56: 26402646, 1985.

7. Schengrund CL and Shochat SJ: Gangliosides in neuroblastomas. Neurochem Pathol 8: 189-202, 1988.

8. Kaucic K, Etue N, LaFleur B, Woods W and Ladisch S: Neuroblastomas of infancy exhibit a characteristic ganglioside pattern. Cancer 91: 785-793, 2001.

9. Hettmer S, Malott C, Woods W, Ladisch S and Kaucic K: Biological stratification of human neuroblastoma by complex B pathway ganglioside expression. Cancer Res 63: 7270-7276, 2003.

10. Wu ZL, Schwartz E, Seeger R and Ladisch S: Expression of GD2 ganglioside by untreated primary human neuroblastomas. Cancer Res 46: 440-443, 1986.

11. Nakamura K, Suzuki M, Taya C, Inagaki F, Yamakawa T and Suzuki A: A sialidase-susceptible ganglioside, IV3 alpha (NeuGc alpha 2-8NeuGc)-Gg4Cer, is a major disialoganglioside in WHT/Ht mouse thymoma and thymocytes. J Biochem 110: 832-841, 1991.

12. Kushi Y, Ogura K, Rokukawa C and Handa S: Blood group Aactive glycosphingolipids analysis by the combination of TLCimmunostaining assay and TLC/SIMS mass spectrometry. J Biochem 107: 685-688, 1990.

13. Ye JN and Cheung NK: A novel O-acetylated ganglioside detected by anti-GD2 monoclonal antibodies. Int J Cancer 50: 197-201, 1992.

14. Sjoberg ER, Powell LD, Klein A and Varki A: Natural ligands of the B cell adhesion molecule CD22 beta can be masked by 9O-acetylation of sialic acids. J Cell Biol 126: 549-562, 1994.

15. Kelm S, Schauer R, Manuguerra JC, Gross HJ and Crocker PR: Modifications of cell surface sialic acids modulate cell adhesion mediated by sialoadhesin and CD22. Glycoconj J 11: 576-585, 1994.

16. Malisan F, Franchi L, Tomassini B, et al: Acetylation suppresses the proapoptotic activity of GD3 ganglioside. J Exp Med 196: 1535-1541, 2002.

17. Mukherjee K, Chava AK, Mandal C, Dey SN, Kniep B, Chandra S and Mandal C: O-acetylation of GD3 prevents its apoptotic effect and promotes survival of lymphoblasts in childhood acute lymphoblastic leukaemia. J Cell Biochem 105: 724-734, 2008.

18. Varki A: Glycosylation changes in cancer. In: Essentials of Glycobiology. Varki A and Cummings R (eds). Cold Spring Harbor Laboratory Press, New York, 1999.

19. Valentino L, Moss T, Olson E, Wang HJ, Elashoff R and Ladisch S: Shed tumor gangliosides and progression of human neuroblastoma. Blood 75: 1564-1567, 1990.

20. Sekiguchi M, Oota T, Sakakibara K, Inui N and Fujii G: Establishment and characterization of a human neuroblastoma cell line in tissue culture. Jpn J Exp Med 49: 67-83, 1979.

21. Miyake S, Shimo T, Kitamura Y, Nojyo T, Nakamura S, Imashuku $\mathrm{S}$ and $\mathrm{Abe} T$ : Characteristics of continuous and functional cell line NB-1, derived from a human neuroblastoma. Autonomic Nervous System 10: 115-120, 1973.

22. Gilbert F, Balaban G, Moorhead P, Bianchi D and Schlesinger H: Abnormalities of chromosome 1p in human neuroblastoma tumors and cell lines. Cancer Genet Cytogenet 7: 33-42, 1982.

23. Tumilowicz JJ, Nichols WW, Cholon JJ and Greene AE: Definition of a continuous human cell line derived from neuroblastoma. Cancer Res 30: 2110-2118, 1970.

24. Schlesinger HR, Gerson JM, Moorhead PS, Maguire H and Hummeler K: Establishment and characterization of human neuroblastoma cell lines. Cancer Res 36: 3094-3100, 1976.

25. Yoshihara T, Ikushima S, Hibi S, Misawa S and Imashuku S: Establishment and characterization of a human neuroblastoma cell line derived from a brain metastatic lesion. Hum Cell 6: 210-217, 1993

26. Biedler JL, Helson L and Spengler BA: Morphology and growth, tumorigenicity, and cytogenetics of human neuroblastoma cells in continuous culture. Cancer Res 33: 2643-2652, 1973. 